\title{
Weak transitivity and agenda control for extended stepladder tournaments
}

\section{Yongjie Yang $^{1}$ (D) Dinko Dimitrov ${ }^{1}$}

Received: 25 November 2019 / Accepted: 6 July 2020 / Published online: 25 August 2020

(c) The Author(s) 2020

\begin{abstract}
A tournament graph over $n$ players is weakly transitive at player $p$ if it contains a Hamiltonian path $\left(p_{1}, p_{2}, \ldots, p_{n}\right)$ with $p_{1}=p$ such that for all odd integers $i \leq n-2$ there is an arc from $p_{i}$ to $p_{i+2}$. We show that weak transitivity at $p$ suffices to make player $p$ win any extended stepladder tournament of degree at most two.
\end{abstract}

Keywords Agenda control · Extended stepladder · Hamiltonian path · Knockout tournament . Weak transitivity

Mathematics Subject Classification C70 · D70 · D71

\section{Introduction}

Knockout tournaments are widely used when it comes to the selection of an alternative from a set of available options. These elimination formats are crucial for collecting important insights in the analysis of electoral systems (cf. Brams and Fishburn 2002; Laslier 1997) and also play a predominant role in sport competitions where one has to determine the winner among a set of players. In such settings it is usually assumed that the tournament organizer possesses reliable information, in the form of a (deterministic) tournament graph over the player set, about who would win the match between every two players. The tournament organizer then specifies a seeding which labels the leaves of a rooted binary tree by players with each internal node being reached by the winner between the two children. The player who reaches the root of the binary tree is the final winner of the knockout tournament.

\footnotetext{
Yongjie Yang

yyongjiecs@gmail.com

Dinko Dimitrov

dinko.dimitrov@mx.uni-saarland.de

1 Chair of Economic Theory, Saarland University, Saarbrücken, Germany
} 
Agenda control has been defined in the work of Bartholdi et al. (1989) as a particular type of manipulation for knockout tournaments. Provided that the tournament organizer has a favorite player, the question of study is whether there exists a seeding in the rooted binary tree which makes this player the final winner of the knockout tournament. This problem has attracted a lot of attention in the literature with the main focus being on balanced rooted binary trees ${ }^{1}$. It is known that the agenda control problem restricted to balanced rooted tree is NP-hard (Aziz et al. 2018). Moreover, the NP-hardness remains even if we further require the tournament organizer's favorite player to be a king ${ }^{2}$ of the tournament graph (cf. Kim and Williams 2015). Strengthening the notion of a king node was shown to allow for efficiently constructible seedings making kings winners in balanced knockout tournaments (cf. Kim et al. 2017; Kim and Williams 2015). We refer the reader to the work of Williams (2016) for an excellent and detailed survey of the recent literature.

A well known necessary condition for a player $p$ to win a knockout tournament is that every node in the tournament graph should be reachable from $p$ via a Hamiltonian path, i.e., there should be a Hamiltonian path in the tournament graph starting at $p$ (cf. Moulin 1988). This condition is also sufficient when the rooted binary tree of the knockout tournament is a stepladder (cf. Williams 2016, Claim 19.6).

In the present paper, we impose the following additional restriction on at least one such path $\left(p_{1}, p_{2}, \ldots, p_{n}\right)$ with $p_{1}=p$ and $n$ being the number of players: for all odd integers $i \leq n-2$ there should be an arc from $p_{i}$ to $p_{i+2}$. Clearly, this kind of weak transitivity "shortens" in a regular way the reachability from $p$ of every node in the tournament graph. We show then in Sect. 3 that weak transitivity at $p$ suffices to make player $p$ win any extended stepladder of degree at most two. This class of knockout tournaments is formally defined and illustrated in Sect. 2, and it includes the standard stepladders for which the mentioned reachability condition has been shown to be crucial. Moreover, its importance has been demonstrated in the work of Arlegi and Dimitrov (2020) when it comes to the design of "fair" knockout tournaments in sports competitions. Using a suitable notion of "fairness", Arlegi and Dimitrov (2020) show that every such knockout tournament has to be an extended stepladder of degree at most two.

\section{Preliminaries}

In this section we reproduce some basic notions from graph theory. For a detailed introduction in the field we refer the reader to the textbooks (Bang-Jensen and Gutin 2008; West 2000).

\footnotetext{
1 A balanced rooted tree is a rooted tree where the levels of the leaves differ at most one. A formal definition is provided in Sect. 2.

2 A node $v$ in a tournament graph is a king if for every other vertex $u$ in the tournament either there is an $\operatorname{arc}$ from $v$ to $u$, or there exists a third node $w$ such that there is an $\operatorname{arc}$ from $v$ to $w$ and an $\operatorname{arc}$ from $w$ to $u$. It is well-known that every tournament has at least one king (cf. Landau 1953). Moreover, the set of all kings in a tournament graph is exactly the so-called uncovered set of the tournament graph (cf. Shepsle and Weingast 1984; Yang and Guo 2017).
} 
Graphs A graph is a tuple $G=(V, E)$, where $V$ denotes a set of nodes and $E$ a set of edges. For ease of exposition, we also use $V(G)$ and $E(G)$ to denote the node set and edge set of $G$, respectively. Two nodes are adjacent if there is an edge between them. The degree of a node is the number of nodes adjacent to it. A graph $G^{\prime}=\left(V^{\prime}, E^{\prime}\right)$ is a subgraph of $G=(V, E)$ if $V^{\prime} \subseteq V$ and $E^{\prime} \subseteq E$. Moreover, if $E^{\prime}=\left\{(u, v) \in E: u, v \in V^{\prime}\right\}$, we say that $G^{\prime}$ is an induced subgraph, or more precisely, a subgraph of $G$ induced by $V^{\prime}$. A simple path (path for short) is a sequence $\left(v_{1}, v_{2}, \ldots, v_{t}\right)$ of nodes such that either $t=1$, or $t \geq 2$ and there is an edge between $v_{i}$ and $v_{i+1}$ for all $i \in\{1,2, \ldots, t-1\}$. The length of $a$ path is defined as the number of edges in the path. A graph is connected if there exists at least one path between every two nodes. A cycle in a graph $G$ is a sequence $\left(v_{1}, v_{2}, \ldots, v_{t}\right)$ of nodes such that $\left(v_{1}, v_{2}, \ldots, v_{t}\right)$ is a path and there is an edge between $v_{1}$ and $v_{t}$ in $G$.

Trees A tree is a connected graph without cycles. A leaf in a tree is a node of degree 1. A nonleaf node is also called an internal node of the tree. It is well-known that between every two nodes there is a unique path in a tree.

A rooted tree is a tree with a specific node called the root. Let $T$ be a rooted tree with root $r$ and with at least two nodes. Let $v \in V(T) \backslash\{r\}$ be a nonroot node, and let $\left(r, v_{1}, v_{2}, \ldots, v_{t}, v\right)$ be the unique path from the root $r$ to the node $v$. Then, the node $v_{t}$ is called the parent of $v$, and consequently $v$ a child of $v_{t}$. Moreover, all nodes except $v$ in the path are called the ancestors of $v$, and $v$ is called the descendant of these nodes. (Note that we do not regard $v$ as a descendant of itself) It is a folklore that for any nonempty subset $L \subseteq V(T) \backslash\{r\}$ of nodes, there is a unique node $v$ such that $v$ is the ancestor of all nodes in $L$ and, moreover, for any child $u$ of $v$ at least one node in $L$ is not the descendant of $u$. This node $v$ is referred to as the least common ancestor of $L$. For a node $v$ in $T$, the subtree of $T$ rooted at $v$, denoted by $T_{v}$, is the rooted tree with root $v$ whose underling graph is the subgraph of $T$ induced by $v$ and all descendants of $v$.

A leaf-to-root path in $T$ is a path from a leaf node to the root. The level of a node $v$ is the length of the unique path from $v$ to the root. Hence, the root has level 0. By $\Gamma(T)$ we denote the set of all leaves of $T$, and $\Gamma(T, \ell)$ the set of all leaves of $T$ of level $\ell$. The height of $T$, denoted by $h(T)$, is the length of a leaf-to-root path with the maximum length. Therefore, it holds that $\Gamma(T)=\bigcup_{\ell=0}^{h(T)} \Gamma(T, \ell)$.

The trunk of $T$ is the path from the root to the least common ancestor of the leaves in the highest level. The distance between a leaf $v$ of $T$ to the trunk of $T$ is the length of the shortest path among all paths between this leaf and the nodes in the trunk. The branch length of $T$ is the maximum distance between leaves and the trunk.

A binary tree is a rooted tree where each internal node has at most two children. A full binary tree is a binary tree so that every internal node has exactly two children. A full binary tree is balanced if the heights of all leaves differ at most one. In a full binary tree, two nodes having the same parent are called siblings. Clearly, every nonroot node in a full binary tree has a sibling.

A stepladder is a full binary tree such that the distance between every leaf to the trunk is exactly one. A full binary tree of branch length at most $q$ is also called an extended stepladder of degree $q$ (cf. Arlegi and Dimitrov 2020). Therefore, stepladders are exactly full binary trees of branch length 1 . We refer to Fig. 1 for an illustration. 


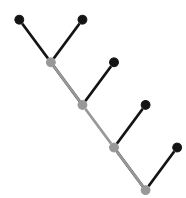

(a)

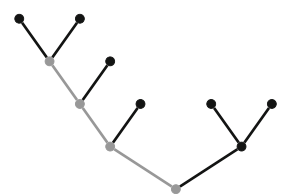

(b)

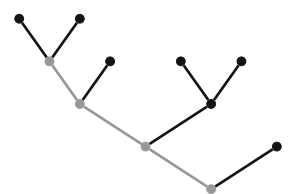

(c)

Fig. 1 An illustration of an extended stepladder of degree 1 (a) and two extended stepladders of degree 2 $(\mathbf{b}, \mathbf{c})$. The trunk in each tree is in gray

Tournament graphs Let $P$ be a set of $n$ players, $n \geq 2$. We assume that information is available about the match outcome (no ties) for every pair of players; that is, for $p \in P$ and $p^{\prime} \in P \backslash\{p\}$, either $p$ beats $p^{\prime}$ or $p^{\prime}$ beats $p$. This information is represented as a tournament graph over $P$, denoted by $G_{P}$. That is, $G_{P}$ is a directed graph with node set $P$ such that, for every two different players $p, p^{\prime} \in P$, there is an $\operatorname{arc}$ from $p$ to $p^{\prime}$ if and only if $p$ beats $p^{\prime}$. A Hamiltonian path in $G_{P}$ is a sequence $\left(p_{1}, p_{2}, \ldots, p_{n}\right)$ of $n$ different nodes such that there is an arc from $p_{i}$ to $p_{i+1}$ for all $i \in\{1,2, \ldots, n-1\}$. Knockout tournaments For a set $P$ of players and a rooted full binary tree $T$ of $|P|$ leaves, a seeding is a one-to-one mapping from $P$ to $\Gamma(T)$. A knockout tournament is a 4-tuple $\left(P, T, \varphi, G_{P}\right)$ where $\varphi$ is a seeding from $P$ to $\Gamma(T)$, and $G_{P}$ is a tournament graph over $P$. Given a knockout tournament $\left(P, T, \varphi, G_{P}\right)$, players reaching internal nodes of $T$ are determined as follows. Initially, a player $p$ occupies a leaf node $v$ if $\varphi(p)=v$. Then, two players occupying two sibling-nodes compete, and the winner moves to the parent node. This procedure continues until some player reaches the root. The corresponding player is then called the final winner of the knockout tournament $\left(P, T, \varphi, G_{P}\right)$.

We use the notion $\left(P^{\prime}, T_{v}, \varphi^{\prime}, G_{P}\left[P^{\prime}\right]\right)$ for the knockout tournament restricted to the subtree of $T$ rooted at node $v$. That is, $P^{\prime}=\left\{p \in P: \varphi(p) \in \Gamma\left(T_{v}\right)\right\}$ is the set of all players seeded to the leaves of $T_{v}$ by $\varphi, \varphi^{\prime}$ is the seeding which maps $P^{\prime}$ to $\Gamma\left(T_{v}\right)$ such that for every $p \in P^{\prime}$ it holds that $\varphi^{\prime}(p)=\varphi(p)$, and $G_{P}\left[P^{\prime}\right]$ is the subtournament of $G_{P}$ induced by $P^{\prime}$.

\section{Weak transitivity and agenda control}

As briefly elaborated in the Introduction, the weak transitivity of a tournament graph strengthens the mentioned necessary condition for making a distinguished player win a given knockout tournament. More precisely, we say that a tournament graph $G_{P}$ is weakly transitive at some player $p_{1}$ if there is a Hamiltonian path $\triangleright=\left(p_{1}, p_{2}, \ldots, p_{n}\right)$ in $G_{P}$ such that for all odd integers $i \leq n-2$ there is an arc from $p_{i}$ to $p_{i+2}$. We call $\triangleright$ the witness of $G_{P}$ at $p_{1}$ in this definition. (Note that if $G_{P}$ is weakly transitive at some player $p_{1}$, it may have more than one witness.) See Fig. 2 for an illustration of a weakly transitive tournament.

Our main result (Theorem 1 ) shows that, provided $T$ is an extended stepladder of degree at most two, weak transitivity of $G_{P}$ at some player $p \in P$ suffices for the existence of a seeding $\varphi: P \rightarrow \Gamma(T)$ making player $p$ the final winner of 


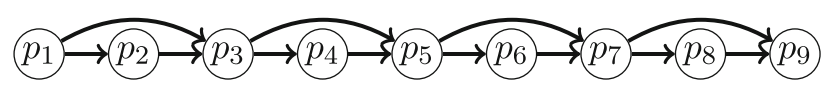

Fig. 2 A weakly transitive tournament. Note that not all arcs are shown. Missing arcs in the tournament can be set arbitrarily. That is, besides the displayed arcs, no additional restrictions are placed on how the other arcs should look like

the knockout tournament $\left(P, T, \varphi, G_{P}\right)$. Before unfolding this result, we present the following useful lemma.

Lemma 1 Let $T$ be a full binary tree with four leaves, $P=\left\{p_{1}, p_{2}, p_{3}, p_{4}\right\}$ a set of four players, and $G_{P}$ a tournament graph over $P$ such that $p_{1}$ beats $p_{2}$ and $p_{3}$, and $p_{3}$ beats $p_{4}$. Then there exists a seeding $\varphi: P \rightarrow \Gamma(T)$ making $p_{1}$ the final winner of $\left(P, T, \varphi, G_{P}\right)$,

Proof There are only two full binary trees with four leaves.

Figure 3 displays these two trees as well as the desired seedings making player $p_{1}$ the final winner of $\left(P, T, \varphi, G_{P}\right)$.

Theorem 1 Let $P$ be a set of players, $G_{P}$ a tournament graph over $P$, and $T$ an extended stepladder of degree at most two with $n=|P|$ leaves. If $G_{P}$ is weakly transitive at some player $p \in P$, then there is a seeding $\varphi: P \rightarrow \Gamma(T)$ making $p$ the final winner of $\left(P, T, \varphi, G_{P}\right)$.

Proof We proceed by induction. The assertion clearly holds when there are two or three players. Due to Lemma 1, the theorem holds when there are four players. Let us now assume that the statement is correct when there are at most $k$ players and show that it also holds when there are $n=k+1$ players, $k \geq 4$.

Let $v$ and $u$ be the two children of the root of $T$ such that $\left|V\left(T_{v}\right)\right| \geq\left|V\left(T_{u}\right)\right|$. In addition, let $\left(p_{1}, p_{2}, \ldots, p_{n}\right)$ be a witness of $G_{P}$, where $p_{1}=p$. So, $p_{1}$ beats both $p_{2}$ and $p_{3}$. Moreover, for each positive integer $i \leq n$, let $P \geq i=\left\{p_{i}, p_{i+1}, \ldots, p_{n}\right\}$. As $T$ has branch length at most two, it suffices to consider the following three cases.

Case 1: There are no leaves in the first level of $T$, i.e., $\Gamma(T, 1)=\emptyset$.

In this case, the subtree $T_{u}$ must contain exactly two leaves and $T_{v}$ contains exactly $n-2$ leaves (see Fig. 4).

By induction, there is a seeding $\varphi^{\prime}$ from $P^{\geq 3}$ to the leaves of $T_{v}$ so that $p_{3}$ is the final winner of $\left(P^{\geq 3}, T_{v}, \varphi^{\prime}, G_{P}\left[P^{\geq 3}\right]\right)$. Then we construct the desired seeding $\varphi: P \rightarrow$ $\Gamma(T)$ based on $\varphi^{\prime}$ as follows:

$-\varphi\left(p_{i}\right)=\varphi^{\prime}\left(p_{i}\right)$ for all $p_{i} \in P^{\geq 3}$ and

- $\left\{\varphi\left(p_{1}\right), \varphi\left(p_{2}\right)\right\}=\Gamma\left(T_{u}, 1\right)$.

As $p_{1}$ beats both $p_{2}$ and $p_{3}$, we know that $p_{1}$ is the final winner of $\left(P, T, \varphi, G_{P}\right)$.

Case 2: There is exactly one leaf in the first and second levels of $T$, respectively, i.e., $|\Gamma(T, 1)|=|\Gamma(T, 2)|=1$.

Let $x$ and $y$ denote the two children of $v$, and without loss of generality, we assume that $\left|V\left(T_{x}\right)\right| \geq\left|V\left(T_{y}\right)\right|$ (see Fig. 5). 

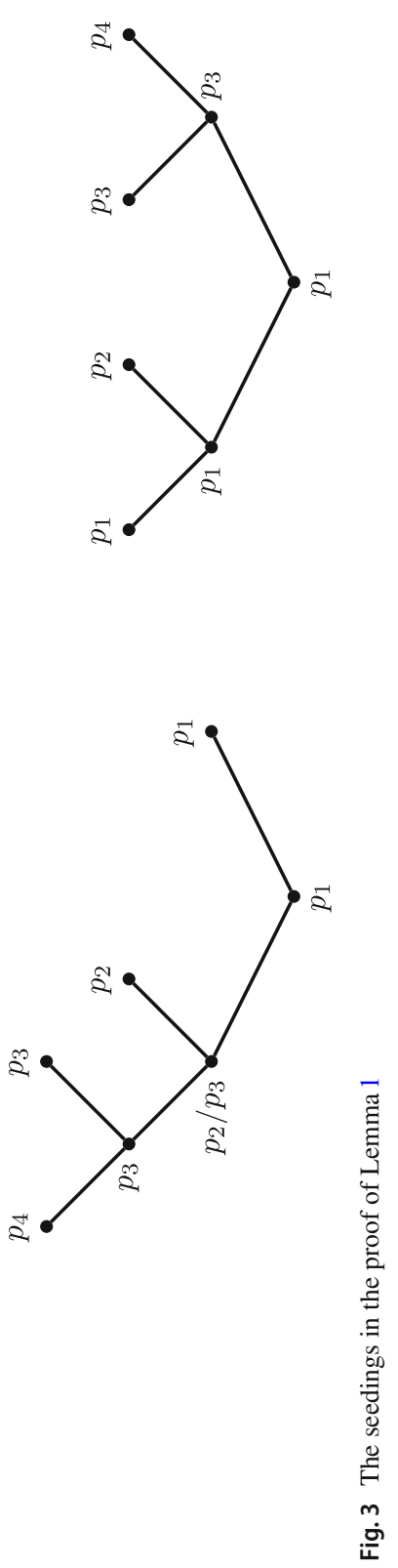
Fig. 4 Illustration of the seeding in Case 1
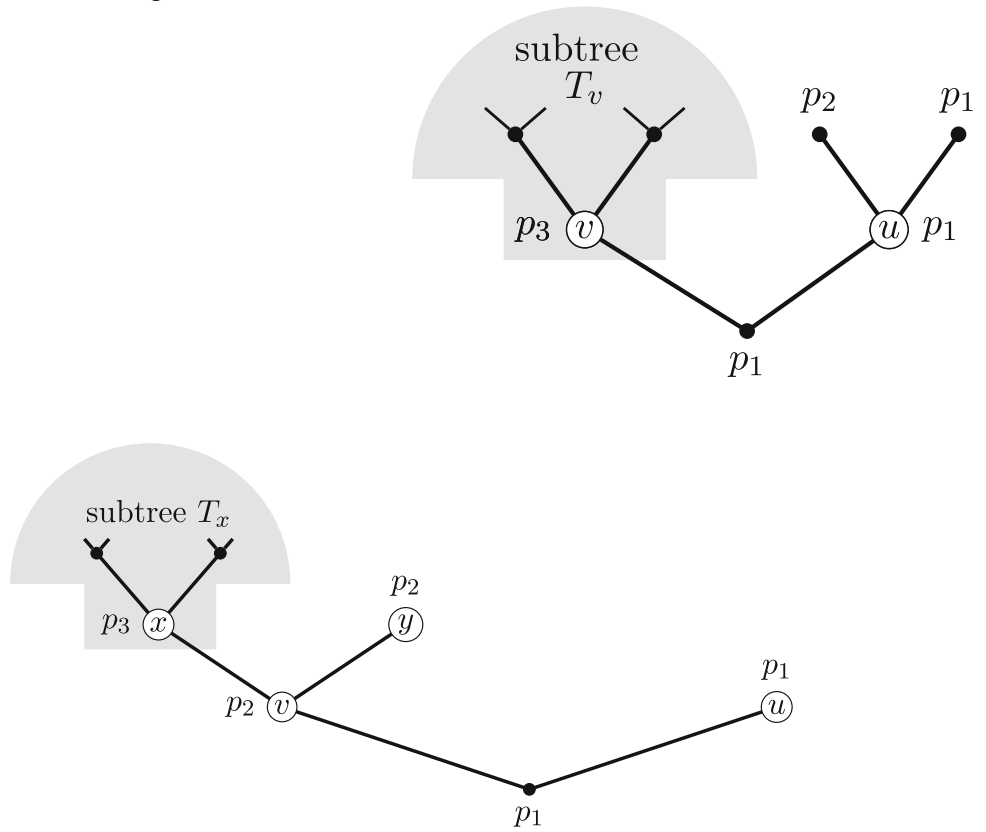

Fig. 5 Illustration of the seeding in Case 2

Due to the induction, there is a seeding $\varphi^{\prime}$ from $P^{\geq 3}$ to $\Gamma\left(T_{x}\right)$ such that $p_{3}$ is the final winner of $\left(P^{\geq 3}, T_{x}, \varphi^{\prime}, G_{P}\left[P^{\geq 3}\right]\right)$. Then we construct the desired seeding $\varphi$ : $P \rightarrow \Gamma(T)$ based on $\varphi^{\prime}$ as follows:

- $\varphi\left(p_{i}\right)=\varphi^{\prime}\left(p_{i}\right)$ for all $p_{i} \in P^{\geq 3}$;

$-\varphi\left(p_{2}\right)=y ;$ and

$-\varphi\left(p_{1}\right)=u$.

It is easy to check that $p_{1}$ is the final winner of $\left(P, T, \varphi, G_{P}\right)$.

Case 3: There is exactly one leaf in the first level, but there are no leaves in the second level, i.e., $|\Gamma(T, 1)|=1$ and $\Gamma(T, 2)=\emptyset$.

We further distinguish between the following two sub-cases.

Case 3.1: $|\Gamma(T, \ell)| \in\{0,2,4\}$ for each $\ell \in\{2,3, \ldots, h(T)\}$.

As $T$ has branch length at most two, it must be that $|\Gamma(T, h(T))|=4$ and $|\Gamma(T, \ell)|=2$ holds for each $\ell \in\{3,4, \ldots, h(T)-1\}$. Letting $t=h(T)-1$, the number of players is then exactly $n=2 t+1$ (odd number). Now we design a seeding $\varphi$ from $P$ to $\Gamma(T)$ as illustrated in Fig. 6 .

The precise definition of the seeding is as follows. We divide the player set $\left\{p_{3}, p_{4}, \ldots, p_{2 t-2}\right\}$ into $t-2$ subsets $P_{3}, P_{5}, \ldots, P_{2 t-3}$ such that each subset $P_{j}$, where $j \in\{3,5, \ldots, 2 t-3\}$, consists of the players $p_{j}$ and $p_{j+1}$. We pair up the players in $P_{3}, P_{4}, \ldots, P_{2 t-3}$, one by one, in the leaves from the ones in the secondlowest level to the ones in the second-highest level, respectively. That is, for each 


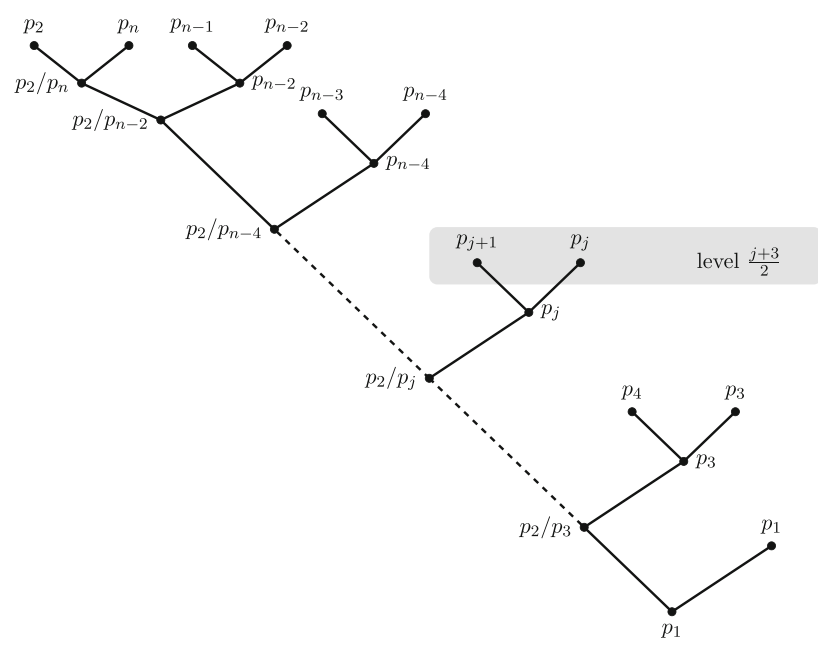

Fig. 6 Illustration of the seeding in Case 3.1

$j \in\{3,5, \ldots, 2 t-3\}$, we let $\left\{\varphi\left(p_{j}, p_{j+1}\right)\right\}=\Gamma\left(T, \frac{j+3}{2}\right)$. Then, we pair up $p_{2}$ and $p_{n}$, and pair up $p_{n-1}$ and $p_{n-2}$ in the highest level leaves. In other words, we set

$$
\left\{\varphi\left(p_{n}\right), \varphi\left(p_{n-1}\right), \varphi\left(p_{n-2}\right), \varphi\left(p_{2}\right)\right\}=\Gamma(T, h(T)),
$$

such that $\varphi\left(p_{2}\right)$ and $\varphi\left(p_{n}\right)$, and $\varphi\left(p_{n-1}\right)$ and $\varphi\left(p_{n-2}\right)$, respectively, are siblings in $T$. Finally, we seed $p_{1}$ to the first level leaf, i.e., $\left\{\varphi\left(p_{1}\right)\right\}=\Gamma(T, 1)$. As $G_{P}$ is weakly transitive at $p_{1}$ with the witness $\left(p_{1}, p_{2}, \ldots, p_{n}\right)$ and $n$ is odd, we know that $p_{n-2}$ beats both $p_{n}$ and $p_{n-1}$. This implies the player reaching the least common ancestor of the leaves in the highest level is either $p_{2}$ or $p_{n-2}$. Moreover, for each subset $P_{j}$, $j \in\{3,5, \ldots, 2 t-3\}$, the player reaching the parent of $\varphi\left(p_{j}\right)$ and $\varphi\left(p_{j+1}\right)$ is $p_{j}$. Again, as $G_{P}$ is weakly transitive at $p_{1}$ with witness $\left(p_{1}, p_{2}, \ldots, p_{n}\right)$, for every $j \in$ $\{3,5, \ldots, 2 t-3\}$, it holds that the player $p_{j}$ beats the player $p_{j+2}$. As a consequence, the player reaching the parent of the parent of $\varphi\left(p_{j}\right)$ for each $j \in\{3,5, \ldots, 2 t-3\}$ is either $p_{2}$ or the player $p_{j}$. In other words, the player reaching a trunk node at level $\ell \geq 1$ is either $p_{2}$ or $p_{2 \ell+1}$. Hence, either $p_{2}$ or $p_{3}$ reaches the second node in the trunk. As $p_{1}$ beats both $p_{2}$ and $p_{3}$, we can conclude that $p_{1}$ is the final winner of $\left(P, T, \varphi, G_{P}\right)$.

Case 3.2: $|\Gamma(T, \ell)|=3$ for some $\ell \in\{3,4, \ldots, h(T)\}$.

Let $i$ be the smallest integer such that in the level $i$ there are exactly three leaves. Let $z$ denote the leave in level $i$ whose parent is in the trunk of $T$. Let $w$ be the sibling of $z$ (which is a trunk node in $T$ ). Obviously, there are exactly $2(i-1)$ leaves of levels at most $i$, i.e., $\left|\bigcup_{\ell=1}^{i} \Gamma(T, \ell)\right|=2(i-1)$. Due to the induction, there is a seeding $\varphi^{\prime}$ from $P^{\geq 2 i-1}$ to the leaves in the subtree rooted at $w$ such that $p_{2 i-1}$ is the final winner of $\left(P^{\geq 2 i-1}, T_{w}, \varphi^{\prime}, G_{P}\left[P^{\geq 2 i-1}\right]\right)$. We design a seeding $\varphi$ from $P$ to $\Gamma(T)$ as follows (see Fig. 7).

First, we seed players in $P^{\geq 2 i-1}$ to the same nodes as in $\varphi^{\prime}$, i.e., $\varphi\left(p_{j}\right)=$ $\varphi^{\prime}\left(p_{j}\right)$ for all $p_{j} \in P^{\geq 2 i-1}$. Then, similar to Case 3.1, we divide the player set 


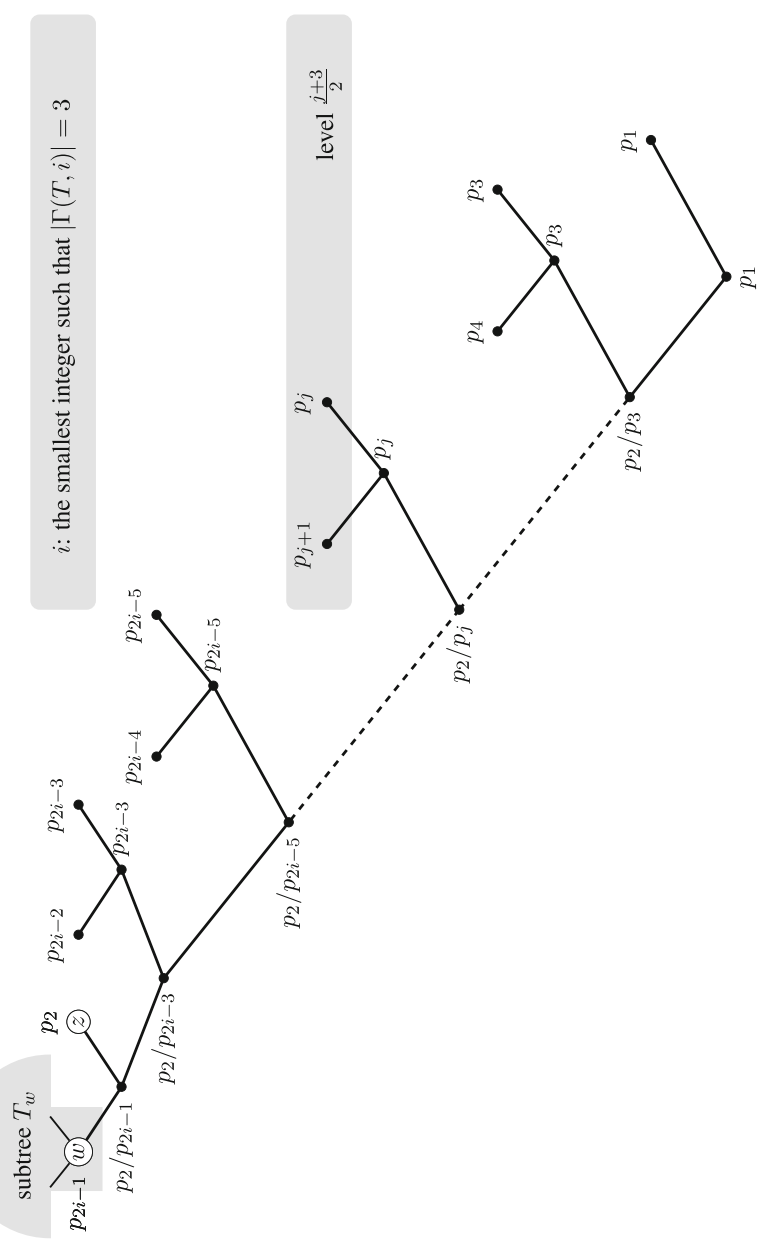

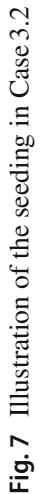




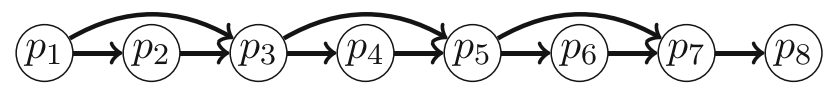

Fig. 8 A tournament graph over eight players. All missing arcs are backwards

$\left\{p_{3}, p_{4}, \ldots, p_{2 i-2}\right\}$ into $i-2$ subsets $P_{3}, P_{5}, \ldots, P_{2 i-3}$ where $P_{j}=\left\{p_{j}, p_{j+1}\right\}$ for each $j \in\{3,5, \ldots, 2 i-3\}$. We seed $p_{1}$ in the first level leaf, and pair up players in each $P_{j}, j \in\{3,5, \ldots, 2 i-3\}$, in the leaves of level $\frac{j+3}{2}$. That is, we let $\left\{\varphi\left(p_{1}\right)\right\}=\Gamma(T, 1)$, let

$$
\left\{\varphi\left(p_{j}\right), \varphi\left(p_{j+1}\right)\right\}=\Gamma\left(T, \frac{j+3}{2}\right) \backslash\{z\}
$$

for all $j \in\{3,5, \ldots, 2 i-3\}$. Finally, we seed $p_{2}$ to the leaf $z$, i.e., $\varphi\left(p_{2}\right)=z$. The player reaching then the parent of $z$ is either $p_{2}$ or $p_{2 i-1}$. Moreover, as $G_{P}$ is weakly transitive with witness $\left(p_{1}, p_{2}, \ldots, p_{n}\right)$, we know that $p_{j}$ beats $p_{j+1}$ and $p_{j+2}$ for all $j \in\{1,3,5, \ldots, 2 i-3\}$. As a consequence, the player reaching the parent of $\varphi\left(p_{j}\right)$ and $\varphi\left(p_{j+1}\right)$ is $p_{j}$ for all $j \in\{3,5, \ldots, 2 i-3\}$. Similar to Case 3.1, we can see that the player reaching a trunk node at level $\ell$, where $\ell \leq i-2$, is either $p_{2}$ or $p_{2 \ell+1}$. Hence, either $p_{2}$ or $p_{3}$ reaches the second node in the trunk. As $p_{1}$ beats both $p_{2}$ and $p_{3}$, we can conclude that $p_{1}$ is the final winner of $\left(P, T, \varphi, G_{P}\right)$.

Remark 1 Notice that the seedings constructed in Lemma 1 and Theorem 1 assign the distinguished player to a leaf in the lowest level of the corresponding rooted binary tree.

Let us finally provide an example showing that Theorem 1 does not extend to full binary trees of branch length at most three. For this, let the player set be $P=\left\{p_{1}, \ldots, p_{8}\right\}$, the tournament graph $G_{P}$ as shown in Fig. 8, and the rooted binary tree $T$ be balanced of eight leaves (and thus an extended stepladder of degree three).

Clearly, $G_{P}$ is weakly transitive at $p_{1}$. Notice that a player has to win three matches in order to reach the root of $T$. Since $p_{1}$ beats only two players in $G_{P}$, we conclude that no seeding in $T$ can make this player the final winner of the tournament.

Acknowledgements Open Access funding provided by Projekt DEAL. The authors would like to thank the editor and the anonymous reviewers for valuable comments that improved the paper.

Open Access This article is licensed under a Creative Commons Attribution 4.0 International License, which permits use, sharing, adaptation, distribution and reproduction in any medium or format, as long as you give appropriate credit to the original author(s) and the source, provide a link to the Creative Commons licence, and indicate if changes were made. The images or other third party material in this article are included in the article's Creative Commons licence, unless indicated otherwise in a credit line to the material. If material is not included in the article's Creative Commons licence and your intended use is not permitted by statutory regulation or exceeds the permitted use, you will need to obtain permission directly from the copyright holder. To view a copy of this licence, visit http://creativecommons.org/licenses/by/4.0/. 


\section{References}

Arlegi, R., Dimitrov, D.: Fair elimination-type competitions. Eur. J. Oper Res 287(2), 528-535 (2020). https://www.sciencedirect.com/science/article/abs/pii/S0377221720302393. Accessed 17 Mar 2020

Aziz, H., Gaspers, S., Mackenzie, S., Mattei, N., Stursberg, P., Walsh, T.: Fixing balanced knockout and double elimination tournaments. Artif. Intell. 262, 1-14 (2018)

Bang-Jensen, J., Gutin, G.: Digraphs: theory algorithms and applications. Springer, London (2008)

Bartholdi, J.J., Tovey, C.A., Trick, M.A.: The computational difficulty of manipulating an election. Soc. Choice Welf. 6(3), 227-241 (1989)

Brams, S.J., Fishburn, P.C.: Voting procedures. In: Arrow, K.J., Sen, A.K., Suzumura, K. (eds.) Handbook of Social Choice and Welfare, pp. 173-236. Elsevier, Amsterdam (2002)

Kim, M.P., Suksompong, W., Williams, V.V.: Who can win a single-elimination tournament? SIAM J. Discrete Math. 31(3), 1751-1764 (2017)

Kim, M.P., Williams, V.V.: Fixing tournaments for kings, chokers, and more. In: Yang, Q., Wooldridge, M.J. (eds.) Proceedings of the Twenty-Fourth International Joint Conference on Artificial Intelligence IJCAI, pp. 561-567. AAAI Press, Palo Alto (2015)

Landau, H.: On dominance relations and the structure of animal societies: III the condition for score structure. Bull. Math. Biophys. 15(2), 143-148 (1953)

Laslier, J.F.: Tournament solutions and majority voting. Springer, Berlin (1997)

Moulin, H.: Axioms of Cooperative Decision Making. Cambridge University Press, Cambridge (1988)

Shepsle, K.A., Weingast, B.R.: Uncovered sets and sophisticated outcomes with implications for agenda institutions. Am. J. Polit. Sci. 28(1), 49-74 (1984)

West, D.B.: Introduction to Graph Theory. Prentice-Hall, Upper Saddle River (2000)

Williams, V.V.: Knockout tournaments. In: Brandt, F., Conitzer, V., Endriss, U., Lang, J., Procaccia, A. (eds.) Handbook on Computational Social Choice, pp. 453-474. Cambridge University Press, Cambridge (2016)

Yang, Y., Guo, J.: Possible winner problems on partial tournaments: a parameterized study. J. Comb. Optim. 33(3), 882-896 (2017)

Publisher's Note Springer Nature remains neutral with regard to jurisdictional claims in published maps and institutional affiliations. 